\title{
Riesgos por COVID-19 durante la gestación, el efecto de la obesidad materna
}

\author{
Risks due to COVID-19 during pregnancy, the effect of maternal obesity \\ Guadalupe López-Rodríguez ${ }^{a}$, Marcos Galván ${ }^{b}$, Rebeca A. Montiel Hernández ${ }^{c}$, Jonathan \\ Alvarez $^{d}$
}

\begin{abstract}
:
Currently, the information about COVID-19, obesity and pregnancy continues to have limitations. At the beginning of epidemic and in accordance with previous experience with other coronaviruses SARS and MERS, poor prognoses were expected for pregnant women with obesity who acquired the disease. Obesity is a chronic and low-grade inflammatory process, which has been associated with worse outcomes during pregnancy and COVID-19. The distribution of visceral adipose tissue is considered as poor prognostic and obese pregnant women have a higher risk of complications. Obese pregnant women have a higher risk of severe complications. The objective of this work was to review the evidence of the effect of obesity and COVID-19 in pregnancy.
\end{abstract}

Keywords:

COVID-19, Pregnancy, Adipose Tissue, Obesity, SARS-CoV-2

Resumen:

Actualmente, la información sobre COVID-19, obesidad y embarazo sigue teniendo limitaciones. Al inicio de la epidemia y de acuerdo con la experiencia previa con otros coronavirus como SARS y MERS, se esperaban un mal pronóstico para mujeres embarazadas con obesidad que adquirían la enfermedad. La obesidad es un proceso inflamatorio crónico y de bajo grado, que se ha visto asociada a peores desenlaces durante el embarazo y COVID-19. La distribución del tejido adiposo visceral tiene el peor pronóstico y las gestantes con obesidad tienen mayor riesgo de complicaciones graves. El objetivo de este trabajo fue revisar la evidencia del efecto de la obesidad y COVID-19 en el embarazo.

\section{Palabras Clave:}

COVID-19, Embarazo, Tejido Adiposo, Obesidad, SARS-CoV-2

\section{Introducción}

Evidencia previa demuestra que el tejido adiposo sirve como reservorio de algunos virus: influenza $A$ y virus de la inmunodeficiencia humana (VIH); se sospecha que también del coronavirus tipo 2 causante del síndrome respiratorio agudo severo (SARS-CoV-2). ${ }^{1}$ Este último responsable de la enfermedad por coronavirus 2019 (COVID-19), para la que se ha reportado mayor riesgo de complicaciones en personas con obesidad.

La obesidad origina un nivel bajo y constante de inflamación como resultado del exceso de moléculas liberadas por los adipocitos y por el resto de las células locales y las que ingresan en el tejido adiposo. ${ }^{2}$ Durante el

Autor de Correspondencia, Universidad Autónoma del Estado de Hidalgo, https://orcid.org/0000-0001-5432-0382, Email: glopez@uaeh.edu.mx

b Universidad Autónoma del Estado de Hidalgo, https://orcid.org/0000-0002-3254-4470, Email: marcos_galvan3112@uaeh.edu.mx

c Universidad Autónoma del Estado de Hidalgo, https://orcid.org/0000-0003-1360-1182, Email: rebecaanahimh@ gmail.com

d Universidad Autónoma del Estado de Hidalgo, https://orcid.org/0000-0002-9434-997X, Email: becarioacademiasnutricion@ gmail.com 
embarazo existe una acumulación fisiológica de tejido adiposo, se ha demostrado que esto genera una respuesta inflamatoria, ${ }^{3}$ misma que incrementa si se tiene obesidad pregestacional. ${ }^{4}$

Previamente se ha señalado una fuerte correlación entre la obesidad y las complicaciones de las infecciones por virus de la influenza y coronavirus anteriores (SARS: síndrome respiratorio agudo grave y MERS: síndrome respiratorio de oriente medio). Ante estas infecciones, se sabe que las mujeres gestantes con sobrepeso u obesidad antes del embarazo tienen un mayor riesgo de morbilidad y mortalidad; el mayor riesgo de mortalidad para estas mujeres se correlacionó con afecciones cardiopulmonares agudas durante el segundo y tercer trimestre de embarazo, asociándose con resultados adversos, incluidos el parto prematuro y la muerte fetal. ${ }^{5}$ Actualmente el SARS-CoV-2, puede causar complicaciones semejantes en la madre y el feto, lo que incrementa el riesgo de sufrimiento fetal, disfunción hepática y muerte materna. ${ }^{6}$

\section{La obesidad como proceso inflamatorio}

El tejido adiposo está conformado por adipocitos, macrófagos, células $\mathrm{T}$, fibroblastos, preadipocitos, células endoteliales, células inmunitarias, etc. Dentro de sus funciones principales está almacenar energía en forma de triglicéridos. ${ }^{7}$ Durante la hiperplasia (división celular) e hipertrofia (aumento de tamaño) de los adipocitos, los macrófagos se introducen en el tejido adiposo, provocando una respuesta inflamatoria. ${ }^{8}$ Durante este proceso se liberan citoquinas como factor de necrosis tumoral a (TNF-a), interleucina 1, 6, 10 (IL-1, IL-6, IL-10, respectivamente); adipocinas como leptina, resistina, adiponectina, proteína quimioatrayente de monocitos 1 (MCP-1), motivo ligando de quimiocinas 5 (CXCL5), angiotensinógeno, moléculas angiogénicas como factor de crecimiento endotelial vascular (VEGF), interferón gamma o alfa (IFN- $\gamma$ o IFN- $\alpha$ ), entre otras. Siendo las principales citoquinas inflamatorias del tejido adiposo el TNF- $\alpha$, IL-1 e IL-6. ${ }^{9}$

\section{Respuesta inflamatoria durante la gestación}

En el embarazo ocurren diversos cambios, entre ellos los de la respuesta inflamatoria materna y del sistema inmunitario innato y adaptativo. ${ }^{10}$ Las principales células del sistema inmunitario innato en el embarazo son macrófagos, células dendríticas y células asesinas naturales (NK), mientras que el adaptativo incluye linfocitos que se activan en presencia de agentes extraños. Ambos participan para que el organismo materno no rechace el feto. ${ }^{11}$ Las células $T$ reguladoras son necesarias para disminuir la inflamación e impiden la generación de células $T$ efectoras que pueden causar un daño al feto. ${ }^{10}$ Durante el embarazo un aumento de neutrófilos e hipersensibilización inmunológica estimula la síntesis de IL-2, IL-6 e IFN- $\alpha$. Por otra parte, las células NK CD56+ y CD16- son necesarias durante el primer trimestre para el desarrollo de la placenta y la tolerancia fetomaterna. ${ }^{12}$ Múltiples mecanismos inmunológicos se modifican durante el embarazo ya que se requieren para mantener un estado de salud adecuado y disminuir el riesgo de complicaciones. ${ }^{13}$

\section{Obesidad y embarazo}

La Encuesta Nacional de Salud y Nutrición (ENSANUT) reportó en 2012 que en mujeres en edad reproductiva la prevalencia de sobrepeso y obesidad era de $35.5 \%$ y 37.5 $\%$, mientras que en $2018-2019$ fue de $36.6 \%$ y $40.2 \%$, respectivamente. ${ }^{14}$ La OMS recomienda que, en mujeres con sobrepeso u obesidad, la ganancia de peso gestacional sea menor a $10 \mathrm{Kg}$, aunque el Instituto de Medicina de EE. UU. sugiere 7 a $11.5 \mathrm{Kg}$ y 5 a $9 \mathrm{Kg}$, respectivamente. ${ }^{15}$ En las mujeres embarazadas con normo peso pregestacional, la correcta ganancia de peso es importante para un pre acondicionamiento fisiológico que demanda el embarazo. ${ }^{2,16}$ Sin embargo, el aumento en el tejido adiposo visceral se relaciona con una mayor producción de proteína $\mathrm{C}$ reactiva $(\mathrm{PCR})$ y citoquinas inflamatorias. ${ }^{17}$ Las mujeres embarazadas con sobrepeso u obesidad y SARS han demostrado tener mayor riesgo de mortalidad y morbilidad, incluyendo patologías cardiopulmonares agudas, principalmente en el segundo y tercer trimestre. ${ }^{5}$

\section{COVID-19 y obesidad}

La infección por SARS-CoV-2 está relacionada con un proceso inflamatorio, provocando deterioro de las células pulmonares. El virus entra a la célula utilizando la enzima convertidora de angiotensina 2 (ACE2) y la serina proteasa TMPRSS2. ${ }^{5}$ Las personas con obesidad son más susceptibles a la infección por SARS-CoV-2, con lo que incrementa la cantidad de citoquinas. ${ }^{18}$ Personas con COVID-19 y obesidad pueden mostrar mayores complicaciones, con una disminución del flujo en las vías respiratorias. ${ }^{19}$

El tejido adiposo visceral tiene funciones endocrinas únicas y secreta diversas adipocinas y citoquinas proinflamatorias.20 Actualmente se considera que la persona con COVID-19 y mayor tejido adiposo visceral tiene peor pronóstico. ${ }^{21}$ Resultados clínicos graves en pacientes infectados por SARS-CoV-2 se deben a un depósito de tejido adiposo visceral elevado; incluso este 
parámetro es mejor que el índice de masa corporal (IMC) para predecir mayores complicaciones por dicho virus. ${ }^{21,22}$

Las primeras investigaciones relacionaron los diagnósticos graves de COVID-19 con el IMC, sin embargo, no se había descrito si la relación era igual para el tejido adiposo subcutáneo (TAS) o visceral (TAV). Actualmente, existe evidencia de que el TAS y TAV se asocian con un mayor riesgo de complicaciones por COVID-19, siendo un parámetro mejor para un diagnóstico grave en comparación al IMC. ${ }^{20}$ Pacientes con grasa visceral y circunferencia abdominal mayores, pueden ser más propensos a requerir tratamiento en la unidad de cuidados intensivos ( $\mathrm{UCl}$ ) y/o intubación para ventilación mecánica. ${ }^{21}$ Un estudio en Alemania reportó que los pacientes que se encontraban en la $\mathrm{UCl}$ presentaban mayor tejido adiposo visceral en comparación con aquellos que se encontraban en otras salas de hospitalización. ${ }^{20}$ Se ha reportado que personas con mayor tejido adiposo visceral tienen más riesgo de diagnósticos graves por COVID-19, siendo más significativo en hombres; incluso tienen menor riesgo factores como la edad y el sexo para diagnósticos formas graves de la enfermedad. ${ }^{22}$

\section{COVID-19, obesidad y embarazo}

Al inició de la epidemia se postuló que en las mujeres gestantes los factores de riesgo para padecer COVID-19 grave eran similares a los de la población general. ${ }^{23} \mathrm{Sin}$ embargo, los desenlaces negativos pueden estar asociados a deficiente atención obstétrica, riesgos sociales y barreras de acceso a servicios de salud. ${ }^{4,24}$ Las mujeres embarazadas presentan complicaciones no solo asociadas a la infección, también a la posible obesidad o comorbilidades previas que se suman con la acumulación fisiológica de tejido adiposo.

Mujeres gestantes con obesidad y COVID-19 pueden tener un riesgo particularmente alto de embolismo pulmonar por condiciones protrombóticas coexistentes. ${ }^{25}$ Durante el embarazo el tamaño del útero incrementa, levantando al diafragma y comprimiendo los pulmones, por lo que una mujer gestante con COVID-19 tiene mayor dificultad respiratoria. Además, el SARS-CoV-2 puede ser un problema para la estabilidad del feto. ${ }^{26}$ Durante el embarazo, la obesidad y sus comorbilidades incrementan el riesgo de desarrollar COVID-19 grave, probablemente por la reducción del volumen respiratorio y la capacidad funcional. ${ }^{27}$

Hasta ahora, los datos clínicos no sugieren resultados diferenciados de mujeres embarazadas infectadas con COVID-19 en comparación con aquellas infectados con
SARS o MERS, ${ }^{26}$ contrario a lo que se especuló en un inicio. A pesar de esto, un estudio en Irán reportó que 7 de cada 9 embarazadas que se infectaron por COVID-19 murieron. ${ }^{28}$ En un reporte de los CDC de Estados Unidos, se informa sobre un mayor porcentaje de obesidad en mujeres embarazadas que ingresan al hospital por causa de COVID-19 (44.2 \%) en comparación con mujeres que ingresaron por otros motivos obstétricos $(30.6 \%) .{ }^{29}$

Una investigación en el estado de Washington reportó una tasa del $15 \%$ de infecciones graves por SARS-CoV-2, ocurrido mayormente en mujeres que presentaban sobrepeso u obesidad antes del embarazo o que tenían comorbilidades como asma. ${ }^{30}$ Según una revisión sistemática, de 30 pacientes que ingresaron a la $\mathrm{UCl}$ (de un total de 322 casos de mujeres embarazadas), tres tenían obesidad como factor de riesgo ante su admisión. ${ }^{31}$ De acuerdo con Barbero et al., la infección por SARSCoV-2 puede no comportarse tan leve como se sugiere durante el embarazo, especialmente al estar presentes factores como obesidad o con origen latinoamericano. ${ }^{32}$ Según Boushra y colaboradores, los datos sugieren que $40 \%$ de las gestantes fallecidas por SARS-CoV-2 presentaban factores de riesgo como obesidad, diabetes o edad mayor de 40 años, aunque admiten que los datos sobre la población embarazada son limitados. ${ }^{33}$

\section{Conclusiones}

La obesidad es un estado de inflamación, que contribuye al desarrollo de múltiples enfermedades, actualmente se considera un factor de riesgo para peores desenlaces por COVID-19 en la población general y durante el embarazo.

Mayor acumulación del tejido adiposo visceral es un buen predictor de resultados clínicos graves y mortalidad por infección de SARS-COV-2 en comparación con otros factores como IMC, la edad y/o sexo.

En las mujeres gestantes que presentan obesidad, su depósito principal de tejido adiposo es el visceral, siendo factor detonante para diagnósticos graves por COVID-19.

Todo indica que las mujeres gestantes con obesidad y COVID-19 tienen mayor riesgo de mortalidad, morbilidad grave, ingreso a la UCI y ventilación mecánica. Si además presentan otras patologías asociadas a la obesidad, se puede agravar el pronóstico.

\section{Referencias}

[1] Huan H, Qingfeng M, Cong L, Rui L, Li Z, Wei W, et al. Profiling serum cytokines in COVID-19 patients reveals IL-6 and IL-10 are 
disease severity predictors. Emerg Microbes Infect. 2020;9(1):1124 30 .

[2] Vega RG, Rico RM. Adipose tissue: immune function and alterations caused by obesity. Rev Alerg Méx. 2019;66(3):340-53.

[3] Ovalle A, Martínez MA, Fuentes A, Marques X, Vargas F, Vergara P, et al. Obesity, a risk factor for ascending bacterial infection during pregnancy. Rev méd Chile. 2016;144(4):476-82.

[4] McCartney SA, Kachikis A, Huebner EM, Walker CL, Chandrasekaran $\mathrm{S}$, Adams WK. Obesity as a contributor to immunopathology in pregnant and non-pregnant adults with COVID-19. Am J Reprod Immunol. 2020;84:13320.

[5] Petrakis D, Margină D, Tsarouhas K, Tekos F, Stan M, Nikitovic D, et al. Obesity a risk factor for increased COVID 19 prevalence, severity and lethality (Review). Mol Med Rep. 2020;22(1):9-19.

[6] Panahi L, Amiri M, Pouy S. Risks of Novel Coronavirus Disease (COVID-19) in Pregnancy; a Narrative Review. Arch Acad Emerg Med. 2020;8(1):34.

[7] Suárez-Carmona W, Sánchez-Oliver AJ, González-Jurado JA Fisiopatología de la obesidad: Perspectiva actual. Rev Chil Nutr 2017;44(3):226-33.

[8] Engin AB. Adipocyte-Macrophage Cross-Talk in Obesity. Adv Exp Med Biol. 2017;960:327-43

[9] Michalakis K, Ilias I. SARS-CoV-2 infection and obesity: Common inflammatory and metabolic aspects. Diabetes Metab Syndr 2020;14(4):469-71.

[10] Robertson SA, Petroff MG, Hunt JS. Immunology of pregnancy. Knobil and Neill's Physiology of Reproduction. 2015:1835-74.

[11] Salazar TL, Ávila GD. Inmunología perinatal. FEMINA 2014;42(4):186-92.

[12] Aghaeepour N, Ganio EA, Mcilwain D, Tsai AS, Tingle M, Van GS, et al. An immune clock of human pregnancy. Sci Immunol 2017;2(15):2946.

[13] Herrera-Pérez JC, Monterio-Fonseca J, Campos-Sánchez S. COVID19 y embarazo. Rev Méd Sinerg. 2020;5(9):492.

[14] Encuesta Nacional de Salud y Nutrición 2018-19. Resultados nacionales. [Available from: https://ensanut.insp.mx/encuestas/ensanut2018/informes.php.

[15] Rasmussen KM, Yaktine AL, Institute of Medicine (U.S.). Committee to Reexamine IOM Pregnancy Weight Guidelines. Weight gain during pregnancy: reexamining the guidelines. Washington, DC: National Academies Press; 2009. xiv, 854 p. p.

[16] Guerendiain M. Impact of maternal obesity on fetal metabolic programming. Latin Perinat. 2016;19(2):152-6.

[17] Mejia MJ, Reyna VE. Obesidad, insulinorresistencia e hipertensión durante el embarazo. Rev Venez Endocrinol Metab. 2017;15(3):169 81.

[18] Stefan N, Birkenfeld AL, Schulze MB, al. e. Obesidad y deterioro de la salud metabólica en pacientes con COVID-19. Nat Rev Endocrinol. 2020;16:341-2.
[19] Yang J, HU J, Zhu C. Obesity aggravates COVID-19: A systematic review and meta-analysis. J Med Virol. 2020;93(1):257-61.

[20] Petersen A, Bressem K, Albrecht J, Thieb H, Vahldiek J, Hamm B, et al. The role of visceral adiposity in the severity of COVID-19: Highlights from a unicenter cross-sectorial pilot study in Germany. Metabolism Clinical and Experimental. 2020;110:317.

[21] Watanabe M, Caruso D, Tuccinardi D, Risi R, Zerunian M, Polici M, et al. Visceral fat shows the strongest association with the need of intensive care in patients with COVID-19. Metabolism. 2020;111:319.

[22] Favre G, Legueult K, Pradier C, Raffaelli C, Ichai C, Iannelli A, et al. Visceral fat is associated to the severity of COVID-19. Metabolism. 2020;115:440

[23] Wastnedge E, Reynolds RM, van Boeckel SR, Stock SJ, Denison FC, Maybin JA, et al. Pregnancy and COVID-19. Physiol Rev. 2021;101(1):303-18.

[24] Takemoto M, Menezes MO, Andreucci CB, Knobel R, Sousa L, Katz $\mathrm{L}$, et al. Clinical characteristics and risk factors for mortality in obstetric patients with severe COVID-19 in Brazil: a surveillance database analysis. BJOG. 2020;127(13):1618-26.

[25] Martinelli I, Ferrazzi E, Ciavarella A, Blasi F, Mosca F, Peyvandi F. Pulmonary embolism in a young pregnant woman with COVID-19. Thrombosis R. 2020;191:36-7.

[26] Hayakawa S, Komine-Aizawa S, Gil GM. Covid-19 pandemic and pregnancy. Obstetrics and Gynaecology Research. 2020;46(10):195866.

[27] Pitones RV, Chávez CE, Hurtado CA, González RA, Serafín HN. Is periodontal disease a risk factor for severe COVID-19 illness? 2020;144:109969.

[28] Hantoushzadeh S, Shamshirsaz AA, Aleyasin A, Seferovic MD, Aski SK, Arian SE, et al. Maternal death due to COVID-19. Am J Obstet Gynecol. 2020;223(1):109 e1- e 16

[29] Panagiotakopoulos L, Myers TR, Gee J, Lipkind HS, Kharbanda EO, Ryan DS, et al. SARS-CoV-2 Infection Among Hospitalized Pregnant Women: Reasons for Admission and Pregnancy Characteristics. CDC. 2020;69(38):1355-9

[30] Thompson LJ, Nguyer ML, Noble NK, Aronoff MD. COVID-19related disease severity in pregnancy. Am J Reprod Immunol. 2020;84:13339.

[31] Novoa RH, Quintana W, Llancarí P, Urbina-Quispe K, Guevara-Ríos E, Ventura W. Maternal clinical characteristics and perinatal outcomes among pregnant women with coronavirus disease 2019. A systematic review. Travel Med Infect Dis. 2020;39:101919.

[32] Barbero P, Muguerza L, Herraiz I, García BA, San Juan R, Forcén L. SARS-CoV-2 in pregnancy: characteristics and outcomes of hospitalized and non-hospitalized women due to COVID-19. J Matern Fetal Neonatal Med. 2020;34(4):30951-7.

[33] Boushra MN, Koyfman A, Long B. COVID-19 in pregnancy and the puerperium: A review for emergency physicians. Am J Emerg Med. 2020;20(30951):735-6757 